\title{
Diferenciais salariais segundo qualificação da força de trabalho e faixa etária no Brasil
}

\author{
Rafael Camargo de Pauli* \\ Luciano Nakabashi* \\ Armando Vaz Sampaio ${ }^{* *}$
}

RESUMO - O processo recente de abertura econômica entre países desenvolvidos e em desenvolvimento foi acompanhado por aumentos nos diferenciais salariais dos trabalhadores qualificados em ambos os grupos de países. Duas teorias principais competem para explicar tais movimentos, o modelo de Heckscher-Ohlin/Stolper-Samuelson e a hipótese do viés de habilidade. $\mathrm{Na}$ tentativa de explicar as mudanças dos diferenciais salariais no Brasil, propõe-se uma adaptação desses modelos para uma economia com três níveis de qualificação. A comparação com os dados empíricos da indústria de transformação posiciona o Brasil como um país abundante em mão-de-obra qualificada, e seus parceiros comerciais como abundantes em mão-de-obra intermediária. Conclui-se que isso se deve ao papel de liderança exercido pelo Brasil no comércio regional, remetendo ao modelo de Davis (1996). Contudo, esses resultados podem estar, em grande parte, sendo influenciados por alguns fatores não incluídos na análise como o aumento da oferta de trabalhadores qualificados e a heterogeneidade não observada da qualificação.

Palavras-chave: Diferenciais salariais. Modelo de Heckscher-Ohlin. Teorema de StolperSamuelson. Hipótese do viés de habilidade. Oferta e demanda por qualificação.

\section{INTRODUÇÃO}

A intensificação da abertura comercial brasileira, a partir do final da década de 1980, foi acompanhada por alterações nos salários relativos de trabalhadores com diferentes níveis de educação. A teoria econômica apresenta duas alternativas principais para explicar essas oscilações. A primeira é constituída pelo modelo de Heckscher-Ohlin (HO), estendido pelo teorema de Stolper-Samuelson (SS). A segunda é a do viés de habilidade. Ambas apontam para sentidos opostos no caso de países em desenvolvimento. Isto é, nesses países, os diferenciais salariais tenderiam a aumentar pelo viés de habilidade e diminuir via HO-SS.

\footnotetext{
* Mestrando em Desenvolvimento Econômico pela UFPR e professor substituto do departamento de Economia da UFPR. Endereço eletrônico: rafaelcdp@gmail.com.

** Doutor em Economia pelo CEDEPLAR/UFMG. Coordenador do boletim Economia \& Tecnologia e professor do Departamento de Economia da UFPR. Endereço eletrônico: luciano.nakabashi@ufpr.br.

*** Doutor em Economia pela ESALQ/USP. Professor do Departamento de Economia (DEPECON-UFPR). Endereço eletrônico: avsampaio@ufpr.br.
} 
De modo a analisar se o Brasil se enquadra em algum padrão de reestruturação salarial proposto pelos modelos descritos acima, este artigo propõe uma adaptação dos mesmos para o caso de economias com o fator trabalho dividido em três níveis de qualificação: qualificado (Q); qualificação intermediária (I); e não-qualificado (NQ). A partir disso, dados empíricos do mercado de trabalho brasileiro são confrontados com os resultados teóricos.

Além dessa introdução, o estudo é dividido da seguinte maneira: na segunda seção são apresentadas as considerações teóricas dos modelos HO-SS e de viés de habilidade, propondo uma adaptação para os mesmos; na terceira seção é realizada a análise empírica e formuladas conclusões preliminares; e, na quarta seção, apresentamos alguns comentários adicionais.

\section{OS MODELOS DE HECKSCHER-OHLIN E DE VIÉS DE HABILIDADE ADAPTADOS}

O modelo HO- SS estabelece que a abertura comercial leva um país a se especializar na produção daquele bem cuja fabricação é intensiva no fator de produção abundante no país. Essa especialização levaria a um deslocamento da produção, aumentando a demanda do fator abundante em detrimento à do fator escasso na economia. Isso afetaria positivamente o preço do primeiro fator em relação ao segundo.

Desse modo, considerando como fatores de produção o trabalho qualificado e o não qualificado, a abertura comercial em países desenvolvidos, que são abundantes em trabalhadores qualificados, aumentaria os salários destes relativamente aos dos trabalhadores menos qualificados. Já no caso de países em desenvolvimento, que possuem abundância em mão-de-obra não qualificada, o nível salarial dos trabalhadores menos qualificados aumentaria em relação ao daqueles com maiores qualificações.

Contudo, estudos empíricos constatam que o hiato salarial entre os trabalhadores qualificados e os não qualificados tem aumentado tanto em economias desenvolvidas quanto em economias em desenvolvimento. ${ }^{39}$ Alguns autores concluem que o modelo de HO-SS é

\footnotetext{
39 Para economias desenvolvidas ver, por exemplo, Sachs e Shatz (1994), Leamer (1996), Haskel e Slaughter, (2001) e Wood (2008), que defendem o modelo de HO-SS para explicar os crescentes diferenciais salariais. Para economias em desenvolvimento ver Arbache, Dickerson e Green (2004).
} 
insuficiente para explicar os movimentos dos mercados de trabalho pelo mundo. A hipótese do viés de habilidade da demanda por trabalho entra em cena a partir disso. ${ }^{40}$

A hipótese do viés de habilidade pressupõe que a abertura comercial, ao possibilitar uma maior difusão tecnológica entre as economias, facilita (barateia) a incorporação de métodos mais eficientes de produção nas firmas, inclusive em países subdesenvolvidos. Esses novos métodos, por sua vez, demandariam mão-de-obra mais qualificada. Por outro lado, a maior concorrência externa forçaria as firmas a reagirem com maior eficiência produtiva, mais qualidade e maior diferenciação do produto, o que, mais uma vez, demandaria mão-de-obra mais qualificada.

Note-se que os dois modelos não são excludentes. Por um lado, ambas as teorias apontam para o mesmo sentido no caso de países desenvolvidos (aumento dos salários relativos dos mais qualificados). Por outro, quando se considera países em desenvolvimento os dois efeitos agem em direção opostas, ou seja, ocorreria uma elevação dos salários relativos dos mais qualificados pela teoria do viés de habilidade, enquanto que pelo modelo HO-SS, o impacto seria de uma redução dos salários relativos dos mais qualificados.

Ainda, é de se esperar que o efeito tecnológico seja proporcionalmente maior em países em desenvolvimento do que nos desenvolvidos, pois existe uma escassez de mão-deobra qualificada naqueles, pressionando para cima os salários dos trabalhadores qualificados de maneira pronunciada. Essa seria uma provável razão para que o efeito líquido do comércio internacional em países em desenvolvimento tenha sido o aumento dos salários relativos dos mais qualificados.

De modo a sistematizar a análise dos impactos da abertura comercial nos salários dos trabalhadores brasileiros, formula-se um quadro demonstrativo com resultados possíveis para os salários relativos no país, segundo classes de qualificação dos empregados. Um fator importante é que os resultados expostos no Quadro 1 não levam em conta oscilações da oferta de trabalho, baseando-se, tão somente, nas considerações dos modelos de HeckscherOhlin e de viés de habilidade. Para o caso brasileiro, a inclusão da oferta de mão-de-obra pode influenciar significativamente as conclusões. Entretanto, tal inserção será realizada pelos autores em trabalhos futuros.

Observe-se ainda, que ao invés de dois fatores - trabalhadores qualificados (Q) e não qualificados (NQ) - como utilizado no modelo de HO, utilizamos três ao incluir os

\footnotetext{
${ }^{40}$ Ver, por exemplo, Berman, Bound e Griliches (1994) e Desjonqueres et al (1999).
} 
trabalhadores com qualificação intermediária (I). A justificativa para isso é encontrada em Andrade e Menezes-Filho (2005, p. 192), onde os autores afirmam que:

Em países desenvolvidos, a maioria dos trabalhadores possui, ao menos, um ano de escolarização secundária, fato que justifica tal divisão [qualificados e não qualificados apenas]. No entanto, em países em desenvolvimento, o nível educacional apresenta uma média bem mais baixa e uma dispersão mais elevada. Uma pequena proporção de trabalhadores possui instrução superior, enquanto uma proporção significativa deles não possui qualquer instrução secundária. Dessa forma, (...) parece ser mais adequado dividir os trabalhadores em mais de dois grupos quando se está conduzindo um estudo (...) para um país em desenvolvimento.

Adicionalmente, supõe-se que existam três níveis salariais, um para cada qualificação, mas que cada país é abundante em apenas um único fator, portanto, o comércio será realizado somente através de produtos intensivos no fator mais abundante e não nos produtos intensivos nos outros fatores, o que implica que só os salários dos trabalhadores abundantes em um e outro país irão variar. Se, por exemplo, o país A tem abundância em Q e o país B abundância em NQ, os salários dos I não vão variar com o comércio entre ambos.

O Quadro 1, expõe as possíveis consequências da abertura do comércio internacional de um países sobre os seus salários relativos, de acordo com a sua abundância de fatores em relação aos seus parceiros comerciais (primeira parte do quadro) e de acordo com a ênfase do viés de habilidade (segunda parte do quadro).

No primeiro resultado, em que ambos os países são abundantes em mão-de-obra não qualificada, o que determina as razões salariais é a diferença salarial do fator NQ entre os países. Se os salários dos NQ domésticos, $n q$, forem maiores do que os salários dos NQ estrangeiros, $n q^{*}$, haverá uma tendência para que eles convirjam. Neste caso $n q$ cai, aumentando i/nq e $q / n q$. Processos semelhantes ocorrem se ambos os países forem abundantes em Q ou se ambos forem abundantes em I.

Já em um caso em que o país doméstico é abundante em mão-de-obra não qualificada e o estrangeiro é abundante em mão-de-obra qualificada, os salários relativos dos qualificados se reduzem em relação aos salários da mão-de-obra intermediária e da NQ, pois a demanda doméstica de Q cairá com a abertura comercial, reduzindo seus salários. A demanda por I não se altera, mas o fato dos salários dos NQ aumentarem faz com que o diferencial dos salários entre intermediários e não qualificados, $i / n q$, também seja reduzido. 
QUADRO 1 - EFEITOS DA ABERTURA COMERCIAL SOBRE OS SALÁRIOS RELATIVOS DOMÉSTICOS

\begin{tabular}{|c|c|c|c|c|}
\hline \multirow{2}{*}{\multicolumn{2}{|c|}{ Heckscher - Ohlin }} & \multicolumn{3}{|c|}{ Fator abundante nos parceiros comerciais } \\
\hline & & NQ & I & $\mathbf{Q}$ \\
\hline \multirow{3}{*}{$\begin{array}{l}\text { Fator abundante doméstico - } \\
\text { NQ }\end{array}$} & $q / i$ & Não altera & Aumenta & Reduz \\
\hline & $i / n q$ & Aumenta se $n q^{*}<n q^{(1)}$ & Reduz & Reduz \\
\hline & $q / n q$ & Aumenta se $n q *<n q$ & Reduz & Reduz \\
\hline \multirow{3}{*}{$\begin{array}{l}\text { Fator abundante doméstico - } \\
\text { I }\end{array}$} & $q / i$ & Reduz & Aumenta se $i^{*}<i$ & Reduz \\
\hline & $i / n q$ & Aumenta & Reduz se $i^{*}<i$ & Aumenta \\
\hline & $q / n q$ & Aumenta & Não altera & Reduz \\
\hline \multirow{3}{*}{$\begin{array}{l}\text { Fator abundante doméstico - } \\
\text { Q }\end{array}$} & $q / i$ & Aumenta & Aumenta & Reduz se $q^{*}<q$ \\
\hline & $i / n q$ & Aumenta & Reduz & Não altera \\
\hline & $q / n q$ & Aumenta & Aumenta & Reduz se $q^{*}<q$ \\
\hline \multirow{2}{*}{\multicolumn{2}{|c|}{ Viés habilidade }} & \multicolumn{3}{|c|}{ Ênfase do viés de habilidade } \\
\hline & & $\mathbf{Q}>\mathbf{I}$ & $\mathbf{I}>\mathbf{Q}$ & $\mathbf{Q}=\mathbf{I}$ \\
\hline & $q / i$ & Aumenta & Reduz & Não altera \\
\hline & $i / n q$ & Aumenta & Aumenta & Aumenta \\
\hline & $q / n q$ & Aumenta & Aumenta & Aumenta \\
\hline
\end{tabular}

FONTE: Elaboração própria.

NOTAS: (1) $x^{*}$ é o salário médio dos parceiros comerciais e $x$ o salário médio doméstico. (2) NQ representa trabalho não qualificado; I representa trabalho com qualificação intermediária; Q representa trabalho qualificado. (3) Os resultados dos salários relativos são referentes àqueles do país doméstico. (4) q/i é o diferencial ou razão entre os salários médios dos qualificados e os salários médios dos intermediários; i/nq é a mesma razão para intermediários e não qualificados e q/nq para qualificados e não qualificados.

Em outro caso, em que o país doméstico seja NQ-intensivo e o estrangeiro Iintensivo, $q / i$ aumenta, apenas em função da queda em $i ; i / n q$ é reduzido em função da queda em $i$ e do aumento em $n q$; e $q / n q$ é reduzido em função do aumento em $n q$, apenas. O mesmo raciocínio é feito para que os demais resultados sejam alcançados, lembrando que os países são abundantes em apenas um único fator e só comercializarão o produto intensivo neste fator.

No caso dos efeitos de viés de habilidade são discriminadas as ênfases desse viés. Caso a tecnologia demandar mais pessoas com nível superior do que aquelas com nível intermediário de educação, então ocorrerá um aumento em $q /$ i e $q / n q$, já o diferencial $i / n q$ tende a aumentar, mas proporcionalmente menos, uma vez que a demanda por qualificação intermediária será menor àquela por trabalhadores qualificados. Caso o viés tecnológico seja favorável à Q e I da mesma maneira, os diferenciais $i / n q$ e $q / n q$ aumentarão e o diferencial $q / i$ tenderá a se manter inalterado. Por fim, caso a ênfase do viés pese mais para a força de trabalho com qualificação intermediária, então $q /$ i apresentará redução e $q / n q$ e $i / n q$ apresentarão aumentos. 
Seria de se esperar que o processo de abertura econômica no Brasil tenha levado à redução nas três razões salariais, $q / \mathrm{i}, q / n q$ e $i / n q$, pois o país possuía na época uma mão-deobra pouco qualificada e se abriu para o comércio com países de mão-de-obra qualificada. A próxima seção verifica essa hipótese.

\section{ANÁLISE EMPÍRICA PARA O BRASIL}

Para verificar se o Brasil se enquadra em uma das situações descritas no Quadro 1, procedeu-se uma análise empírica através de dados dos salários dos trabalhadores brasileiros no período de 1985 a 2007 extraídos da Relação Anual de Informações Sociais (RAIS).

Com base em Fernandes e Menezes-Filho (2001), empregamos os dados dos salários médios dos trabalhadores separados por idades entre 25 a 29 anos, 30 a 39 anos e 40 a 49 anos. Isso se justifica, pois as faixas etárias mais velhas apresentam maior estabilidade no emprego, o que pode levar as mesmas a apresentarem resultados diferentes daqueles dos mais jovens.

Adicionalmente, com base em Andrade e Menezes-Filho (2005), a amostra consistiu somente daqueles trabalhadores que tinham o quinto ano do ensino fundamental completo (classificados como não qualificados), o ensino médio completo (classificados como intermediários) e com ensino superior completo (classificados como qualificados), o que resultou em uma amostra anual média de 12 milhões de empregados. ${ }^{41}$ Esse critério de seleção das escolaridades se deve ao fato de que se todas elas fossem consideradas os salários dos trabalhadores apresentariam variância muito elevada, o que poderia comprometer a análise dos dados. Não obstante, a maior parte dos trabalhadores declarados na RAIS tem escolaridades completas, o que faz com que a amostra seja bastante representativa da população.

Os diferenciais salariais entre as categorias de trabalhadores foram formulados através do cálculo do logaritmo natural das razões dos salários médios (ou diferença dos logaritmos das médias salariais) entre qualificados e não qualificados, qualificados e intermediários e intermediários e não qualificados, separados por faixas de idade. Os resultados estão expostos no Gráfico 1, a seguir.

${ }^{41}$ Esses autores classificam como não qualificados aqueles que têm 4 anos de estudo, intermediários aqueles com 11 anos de estudo e qualificados aqueles com 15 anos de estudo. A base foi construída com os microdados da PNAD. 
Pelas figuras apresentadas observamos que os diferenciais $q / i$ cresceram ao longo de todo o período, para todos os setores, sendo que esses diferenciais se elevaram, principalmente, a partir de 1994-98. Observa-se também que esses diferenciais para os mais jovens (linhas contínuas mais finas) sofreram redução no final do período. Por outro lado, os diferenciais $q / i$ são os que apresentam a menor dispersão entre as faixas etárias (isso se vê, pois as linhas contínuas estão mais unidas do que as tracejadas e as pontilhadas). Isso pode estar indicando que a experiência no trabalho é menos relevante para os dois grupos de trabalhadores em questão e que essa característica não foi alterada com a abertura.

Em relação aos diferenciais $i / n q$, observamos que ocorreram quedas em todos os setores. Estas são pronunciadas para a indústria de transformação, a partir de 2003, e para os demais setores, a partir de 1996-99. A dispersão entre as faixas etárias é bastante elevada nesses casos, sobretudo nos serviços e na agropecuária. Em todos os setores, os diferenciais dos mais jovens foram sempre os menores, mas todas as faixas etárias apresentaram tendências declinantes.

O diferencial $q / n q$, por sua vez, apresentou comportamentos distintos entre os setores. $\mathrm{Na}$ indústria houve crescimento, a partir de 1994. Não obstante, para os mais jovens, essa tendência se inverteu, a partir de 2001. No comércio a tendência foi parecida, mas menos intensa. Nos serviços, apesar de constantes oscilações iniciais, os diferenciais para todas as faixas etárias não mudaram muito entre 1994 a 2007. Já a agropecuária, que entre 1992 e 1994 apresentou crescimentos importantes nesse diferencial, a partir de 1999, registrou quedas em todas as faixas etárias. 
GRÁFICO 1 - DIFERENCIAIS SALARIAIS MÉDIOS POR CLASSE DE QUALIFICAÇÃO E FAIXA ETÁRIA EM QUATRO SETORES DA ECONOMIA BRASILEIRA - 1985- 2007
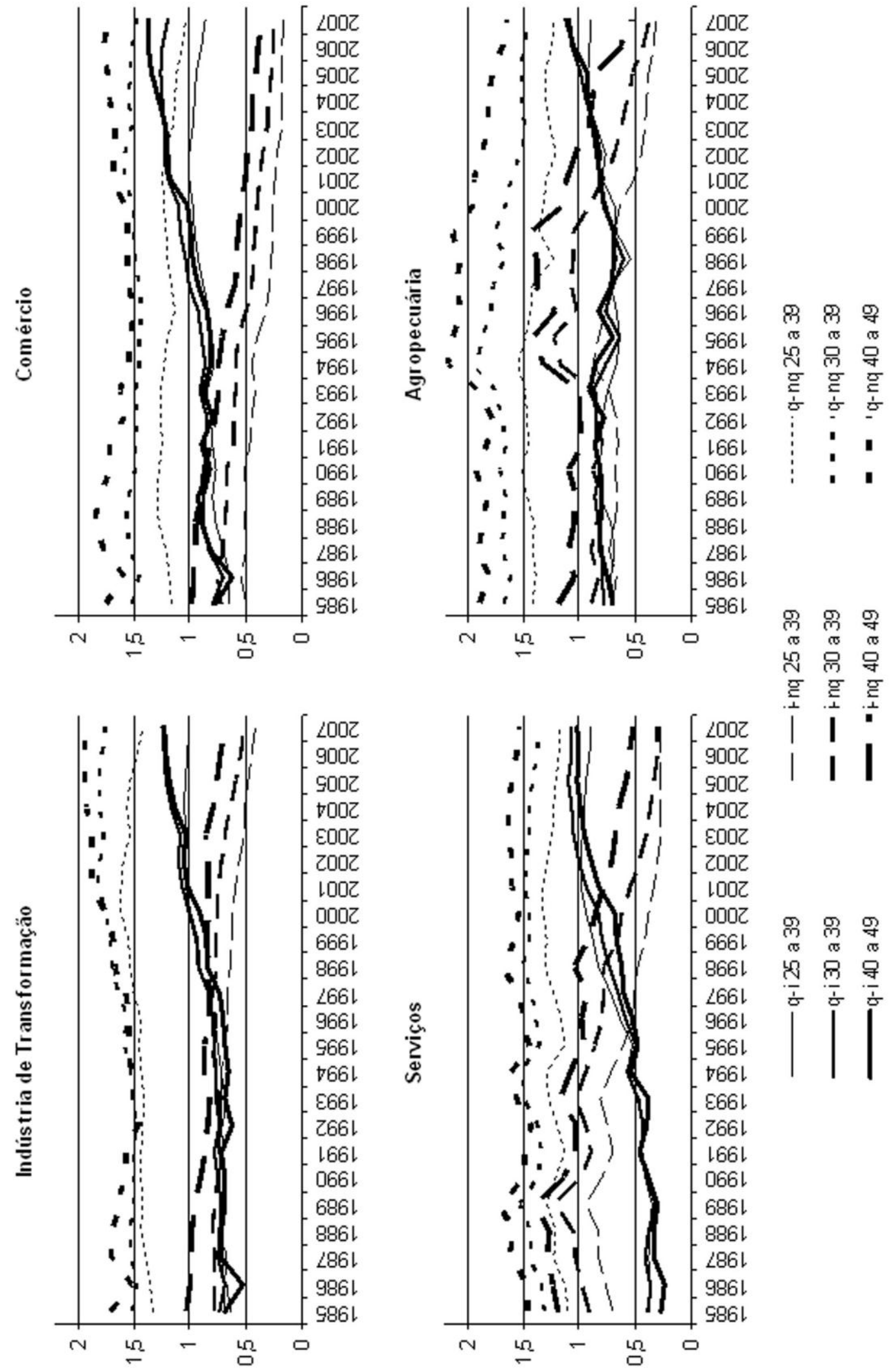

FONTE: Elaboração própria. Dados da Rais. 
Especificamente em relação à indústria de transformação ${ }^{42}$, o Gráfico 1 mostra que, a partir da metade da década de 1990, os diferenciais salariais médios $q / n q$ e $q / i$ sofreram elevações (sobretudo para os mais velhos), enquanto $i / n q$ sofreu redução. Nesse caso, de acordo com a sistematização apresentada no Quadro 1, o Brasil se enquadraria em uma situação em que houvesse neste abundância de mão-de-obra qualificada, com parceiros comerciais abundantes em mão-de-obra com qualificação intermediária. Se considerarmos que a maior parte das exportações da indústria de transformação brasileira, com maior coeficiente tecnológico, tem como destino os países da América do Sul e que estes possuem setores industriais menos competitivos que o brasileiro e, portanto, com mão-de-obra empregada na indústria menos qualificada que a brasileira, essa hipótese seria plausível.

Dados do Aliceweb/ MDIC mostram que entre 1996 e 2008, em torno de 45\% das exportações brasileiras destinadas a países da América Latina e Caribe foram constituídas de bens das seções XVI, XVII, XVIII e XIX, da Nomenclatura Comum do Mercosul (NCM), caracterizadas por produtos manufaturados de alto valor agregado, como veículos automotores, aeronaves, máquinas e aparelhos elétricos, etc. No mesmo período, do total de exportações registradas nessas seções, também cerca de 45\% tinham como destino países da AL e Caribe.

Em outras palavras, a mão-de-obra no Brasil poderia ser considerada qualificada pelos padrões regionais e, portanto, a intensificação do comércio com países vizinhos teria um efeito positivo nos salários dos trabalhadores qualificados. Nesse sentido, Davis (1996) mostra que países que são trabalho intensivo para os padrões internacionais podem apresentar aumentos nos diferenciais salariais dos mais qualificados após um processo de liberalização, se eles forem abundantes em capital em nível regional. ${ }^{43}$

Mesmo desconsiderando o fato de que o Brasil possua uma maior abundância de pessoal qualificado do que seus países latino americanos, deve-se considerar que o país foi um dos últimos da região (e a última grande economia da região) a promover a abertura comercial e que essa abertura foi muito menos traumática do que na maioria dos seus vizinhos (pelo menos em relação à queda das barreiras comerciais). Desse modo a abertura comercial no Brasil não desmantelou a indústria do país como o fez em países semelhantes da região como

\footnotetext{
${ }^{42}$ Esse setor merece destaque, pois está mais suscetível aos impactos diretos do mercado internacional do que os setores comércio e serviços, por exemplo.

43 A abundância de capital tende a ser diretamente correlacionada com a abundância de mão-de-obra qualificada. É o que se denomina na literatura de Complementaridade entre capital e habilidade. Ver Griliches (1967) e Krusell et al. (1997).
} 
Argentina e Uruguai. Isso teria dado margem para que o país se tornasse um líder regional nas exportações de bens industrializados de maior valor agregado. Essa liderança regional teria um impacto positivo nos salários dos trabalhadores qualificados da indústria de transformação do país, maior do que o impacto negativo que possivelmente foi provocado pelo comércio com países desenvolvidos.

Por outro lado, as tendências dos diferenciais salariais não são condizentes com os resultados da hipótese do viés de habilidade, apresentados no Quadro 1. Supondo que os mais jovens são mais aptos para absorver novos conhecimentos tecnológicos, seus diferenciais de salários $q / n q$ e $q / i$ deveriam acompanhar (ou superar) os dos mais velhos, o que não ocorre na indústria de transformação.

Conclui-se, preliminarmente, que o processo de abertura econômica brasileiro beneficiou os trabalhadores mais qualificados do setor industrial, na medida em que grande parte do comércio promovido com a abertura foi realizado entre países da América Latina, região na qual o Brasil se sobressai, com algumas exceções, em relação à disponibilidade de mão-de-obra relativamente mais qualificada.

\section{CONCLUSÃO}

De acordo com dados da RAIS, o processo de abertura comercial no Brasil foi acompanhado por aumentos nos diferenciais de salários $q / i$ e $q / n q$ e redução em $i / n q$, na indústria de transformação. Esses resultados confrontados com uma construção teórica baseada nos modelos HO-SS e no de viés de habilidade, apontam o primeiro como sendo o que explicaria o padrão de comportamento dos salários relativos no país. Ainda, esse padrão aponta o país como sendo abundante em mão-de-obra qualificada e seus parceiros abundantes em mão-de-obra com qualificação intermediária. Esse resultado pode ser admissível, uma vez que a indústria brasileira tem desempenhado um papel de liderança regional, sobretudo no comércio com os parceiros do Mercosul.

Contudo, reconhece-se que a análise não inclui uma série de fatores que poderiam estar influenciando os resultados e, portanto, levando a conclusões tendenciosas. O primeiro deles é o de que o modelo de HO-SS é de longo prazo e que a estrutura salarial brasileira ainda não tenha se acomodado à nova realidade da abertura comercial. Duas outras importantes omissões são a heterogeneidade não observada da qualificação dos trabalhadores e o grande aumento da oferta de trabalhadores com escolaridade média completa devido a 
fatores institucionais, sobretudo a partir de 2002. A análise desses fatores e um maior grau de sofisticação no tratamento dos dados serão deixados para pesquisas futuras.

\section{REFERÊNCIAS}

Análise de Informações de Comércio Exterior (ALICE-Web). Brasília: MDIC. Disponível em: http://aliceweb.desenvolvimento.gov.br.

ANDRADE, A. A. S.; MENEZES-FILHO, N. A. (2005). O papel da oferta de trabalho no comportamento dos Retornos à educação no brasil. Pesquisa e Planejamento Econômico, v.35, n.2.

ARBACHE, J. S.; DICKERSON, A.; GREEN, F. (2003). Trade liberalization and wages in developing countries. Disponível em: http://papers.ssrn.com/sol3/papers.cfm?abstract_id= 447141.

BERMAN, E. J.; BOUND, J.; GRILICHES, Z. (1994). Changes in the demand for skilled labour within U.S. manufacturing: evidence from the Annual Survey of Manufacturers. Quarterly Journal of Economics. vol. 109.

DAVIS, D. R. (1996). Trade Liberalization and Income Distribution. NBER Working Paper No. W5693. Disponível em: http://papers.ssrn.com/sol3/papers.cfm?abstract_id=3491.

DESJONQUERES, T.; MACHIN, S.; VAN REENEN, J. (1999). Another nail in the coffin? Or can the trade based explanation of changing skill structures be resurrected? Scandinavian Journal of Economics,vol. 101.

FERNANDES, R..; MENEZES-FILHO, N. A. (2002). Escolaridade e demanda relativa por trabalho: uma avaliação para o Brasil nas décadas de 80 e 90. São Paulo: USP, 2001, mimeo.

GRILICHES, Z. (1967). Production Functions in Manufacturing: Some Preliminary Results, in The Theory and Empirical Analysis of Production. NBER Studies in Income and Wealth, 31, New York: Columbia University Press.

HASKEL, J.; SLAUGHTER, M. J. (2001). Trade, technology and UK wage inequality. Economic Journal, vol. 111.

KRUSELL, P.; OHANIAN, L. E.; RIOS-RULL, J. V.; VIOLANTE, G. L. (1997). CapitalSkill Complementarity and Inequality: A Macroeconomic Analysis. Federal Reserve Bank of Minneapolis. Staff Report n. 239. Disponível em: http://ideas.repec.org/p/fip/fedmsr/239.html.

LEAMER, E. E. (1996). Wage inequality from international competition and technological change: theory and country experience. American Economic Review, vol. 86. 
RELAÇÃO ANNUAL DE INFORMAÇÕES SOCIAIS (RAIS). Brasilia: MTE. Disponivel em:

www.mte.gov.br.

SACHS, J. D.; SHATZ, H. J. (1994). Trade and jobs in U.S. manufacturing. Brookings Papers on Economic Activity, vol. 1.

WOOD, A. (1995). How Trade Hurt Unskilled Workers.. The Journal of Economic Perspectives, Vol. 9, No. 3. 